\section{Human Trials: Scientists, Investors, and Patients in the Quest for a Cure} for a Cure by Susan Quinn. This is the story of Howard Weiner, a neurologist on the faculty of Harvard Medical School and an attending physician at the Brigham and Women's Hospital. Although different members of Weiner's lab and executives of AutoImmune Inc., Weiner's biotech company, characterized in 1946 by Merrill Chase, who reported that feeding allergens to guinea pigs diminished immune responses and created tolerance to subsequent injections of the allergens. In the mid to late 1980 s, several researchers, including Weiner, began to apply Chase's idea of oral tolerance to animal models of autoimmune disorders. Enter the venture capitalists, captivated by the simple and appealing idea (especially in an era of growing faith in alternative medicine) of curing disabling autoimmune diseases, from MS and arthritis to diabetes, by taking a protein pill with almost no side effects. The investors created a company, recruited executives, supported research in Weiner's lab and began planning for clinical trials of oral tolerance in humans. is long on description and narrative and make appearances, some as brief cameos, Quinn's sympathetic and deferential focus is on Weiner. She traces her subject from his childhood in Denver to his training in neurology, from his struggles as a research fellow in the virology lab of Bernard Fields to his dedication to multiple sclerosis (MS), and from his research laboratory to the development of his biotechnology company. The most appealing aspect of this portrait is Weiner's commitment and concern for his fellows and trainees and their reciprocal affection, loyalty and praise for Weiner. One fellow calls him a father figure, and others say they "owed all [their] success to him."

Overall, Human Trials is a well-told and occasionally gripping story, with a lucid, linear narrative. For laypeople unfamiliar with clinical trials, it serves as an intelligible introduction. The heart of the story concerns Weiner's re-discovery of oral tolerance and how attempts to harness it to treat MS and rheumatoid arthritis led to the founding of a biotech company, AutoImmune. Oral tolerance was first Although the good story line is the book's virtue, it is also its defect. The book short on analysis and evaluation. Its descriptions skirt or simply fail to explore the serious ethical issues re-

homie between Weiner and Bishop are tenderly described, no serious treatment is attempted of the ethics or wisdom of a Harvard professor spending 18 continuous days wooing investors, and the countless other days devoted to work at the biotechnology company. Quinn's version of the Weiner-AutoImmune story is not fashioned to examine how corporate interests and funding influence, distortand maybe even corrupt - the training of research fellows, the agenda of academic researchers, the integrity of research data and the dissemination of research results.

If there were problems in the WeinerAutoImmune relationship, clear insights into this relationship would allow readers to better understand the challenges currently posed by the ever-closer linking of industry to academic medical researchers. Equally, it is possible that Weiner and AutoImmune managed to avoid the ethical pitfalls of industry-academic links, and this case could serve as a 'best practice' for others to learn from and emulate as they confront similar problems.

Unfortunately, Quinn's story is an admiring personality profile facilitated by free access to her subjects, including even Weiner's personal diary. Human Trials simply does not tell us enough to help us really understand the nuances of financial support by pharmaceutical and biotechnology companies and venture capitalists' investments in academic medical research, assess their positive and negative ramifications or determine how to safeguard the values and virtues of academic medical centers.

capital and prominent academic institutions. To be sure, there is whispered acknowledgment that the potential impact on AutoImmune's stock price might have affected the selection of the journals in which to publish, and questions are raised about the appropriateness of jumping to a Phase III randomized trial in MS when oral tolerance had been tried on only a handful of patients, without ever having determined a 'correct' dose. But much more is missing. Quinn writes, "because AutoImmune was cutting back to bare bones, the company could no longer underwrite much research in the [Weiner] lab." Nothing more is said of the ethical concerns raised by the apparent co-mingling of the company and Weiner's Harvard research group. Indeed, that this might be a serious concern does not even seem to occur to Quinn. One chapter describes the 'road show' by Weiner and AutoImmune's CEO, Bob Bishop, to raise investor interest. While Weiner's videotaping of the road show and the bon-

\section{Poverty, Inequality and Health: An International Perspective}

\author{
Edited by David Leon and Gill Walt \\ Oxford University Press, 358 pp, $\$ 55.00$ \\ ISBN: 0192631969; 2001 \\ REVIEWED BY VICTOR W. SIDEL \\ Montefiore Medical Center and \\ Weill Medical College of Cornell University \\ New York, New York, USA
}

The strongly negative correlation between poverty and health has long been known. Poverty and social and economic inequality have been clearly demonstrated to be 